\title{
The link of inflammation and neurodegeneration in progressive multiple sclerosis
}

\author{
Fernando Pérez-Cerdá, María Victoria Sánchez-Gómez and Carlos Matute
}

\begin{abstract}
Progressive multiple sclerosis (MS) is characterized clinically by the accumulation of neurological disability without unequivocal recovery. Understanding the mechanisms that determine entering in this stage of the disease is a great challenge in order to identify potential therapeutic targets. Recent advances in defining more accurately the progressive phenotype of MS, have concluded that differences between primary and secondary progressive forms of disease are relatively quantitative rather than qualitative. In both cases, a large number of molecular and cellular events that might lead to neurodegeneration have been suggested. These include microglia activation, chronic oxidative injury, accumulation of mitochondrial damage in axons, age-related disturbances and dysfunctional axonal transport among others. Commonly, these pathological mechanisms have been considered as a result of inflammatory demyelination but a primary degenerative condition has also been argued. It is now clear that both events contribute to the progression of the disease, however their temporal sequence is still a matter of debate. A detailed knowledge of progressive MS pathogenesis will allow to develop effective treatments for both progression and symptom management that should be based on a combination of anti-inflammatory, regenerative and neuroprotective strategies. In this review, we summarize current data and recent hypothesis about pathological forces that drive progression of damage in MS, i.e. cumulative cortical demyelination and neurodegeneration as well as diffuse alterations (microglia activation, axonal injury and atrophy) throughout white and grey matter in the brain and spinal cord. Finally, we discuss the potential of the aforementioned proposed disease mechanisms with regard to developing suitable therapies to halt the progression in MS pathology.
\end{abstract}

Keywords: Inflammation, Microgliosis, Mitochondria, Energy failure, Axonal damage, Iron accumulation

\section{Background}

Multiple sclerosis (MS) is a chronic inflammatory, demyelinating and neurodegenerative disorder of the central nervous system (CNS) and it is the most common cause of non-traumatic neurological disability in young adults [1-7]. The diagnostic criteria for MS are the clinical course of disease supported by paraclinical laboratory assessments and the demonstration by magnetic resonance imaging (MRI) of dissemination of lesions in space and time. The initial clinical phenotype of MS is frequently characterized by episodes of neurological disturbances followed with residual deficits or full recovery (relapsingremitting MS, RRMS) and in a minority $(10-20 \%)$ by a

\footnotetext{
* Correspondence: fernando.perez@ehu.eus

Achucarro Basque Center for Neuroscience, Departamento de Neurociencias and CIBERNED, Universidad del País Vasco (UPV/EHU), 48940 Leioa, Spain
}

slowly accumulation of disability from the onset (primary progressive MS, PPMS). Usually with time, a majority of RRMS patients (up to $70 \%$ ) transitions into a predominant gradual worsening over exacerbations or relapses (secondary progressive MS, SPMS) [8].

Recently, the classification of the MS phenotypes has been reorganized into categories according to the presence/ absence of activity and/or gradual illness progression [9]. Disease activity is defined by clinical relapses and/or lesion activity in CNS imaging and is related to episodes of tissue injury associated with inflammation. Progression is linked to increasing neurologic dysfunction which by current understanding reflects neurodegenerative processes. Progression and activity are very close to one another but conceptually four phenotypes are possible: progression with/without activity; no progression but with activity; 
and no progression and no activity (stable disease). Thus, SPMS and PPMS are considered parts of the spectrum of progressive MS (PMS) phenotypes and differences between them are relative rather than absolute. Almost no treatment options are available for PMS patients (more than $50 \%$ of people with MS), so a good clinical classification of patients is very important to better design trials for study the value of new therapies $[10,11]$. However, the most fundamental issue in developing new treatments for PMS is to understand the pathological events that underpin the disease process.

The pathological mechanisms that drive neurodegeneration in PMS are poorly understood though a large range of disease processes have been proposed as discussed here. Commonly, neurodegeneration has been regarded as a result of inflammatory demyelination due to peripheral immune system activation (the outside-in hypothesis). Recently an explanation of disease progression suggests that inflammatory demyelinating processes in early MS trigger a cascade of events (among others microglia activation, chronic oxidative injury, mitochondrial damage in axons) that lead to neurodegeneration and are amplified by pathogenic mechanisms related to brain ageing and accumulated disease burden [2, 12-14]. Alternatively, MS can be regarded as a primary degenerative condition which initiates in the myelinating unit (oligodendroglia, their processes and myelin) and results in neuroinflammation (the inside-out hypothesis) $[15,16]$. It is highly likely that immune-triggered inflammation in turn drives further damage and degeneration of CNS elements, creating a vicious circle. According to this hypothesis, progression in MS would be present from the beginning of illness and progressive cytodegeneration would underlie all disease processes. Resolution of these conflicting ideas is unresolved, nonetheless both suggest that treatment of PMS, preferable at the early stages of disease, should be based on a combination of antiinflammatory, regenerative and neuroprotective strategies $[11,17,18]$. Nevertheless, although inflammatory and neurodegenerative events act in concert to induce MSspecific brain damage their relevance changes during the course of chronic disease evolution.

\section{Review}

The pathology of MS is defined by a spectrum of tissue alterations in the CNS $[2,6,19]$. Classically include plaques of primary demyelination with oligodendrocyte loss and profound axonal degeneration surrounded by an astrocytic scar formation in the white and gray matter of the brain and spinal cord. These plaques are typically inactive and very frequent in all stages of MS. In contrast, active lesions display a focal disruption of the bloodbrain-barrier (BBB) together with inflammatory cells (lymphocytes, macrophages and activated microglia) which are present throughout the lesion or at the periphery in acute or chronic active plaques respectively. In PMS half of focal plaques are characterized by a peripheral small rim composed of a small number of inflammatory cells (slowly expanding pre-existing lesions) whereas damage to BBB is less obvious. In addition to focal plaques, diffuse alterations affecting the normal-appearing CNS with a low-grade on-going inflammation are seen in patients with PMS (Table 1). These changes include microglial activation mild demyelination and axonal-neuronal loss in the context of an inflammatory process partly compartmentalized behind an intact BBB.

There is an increasing recognition in the MS field $[6,12,14,19-22]$ that progression of disease that finally result in CNS atrophy, is related with the accumulation over time of the following lesions:

- grey matter demyelination, particularly with a subpial location, largely irrespective of immune cell infiltration and lesion load in white matter tracts; and that is associated with both a very limited remyelination capacity and destruction and loss of nerve cells.

- diffuse tissue loss in the normal-appearing CNS, that are only weakly correlated with the number, size and destructive changes in classical focal white matter lesions.

Assuming that SPMS and PPMS share the same pathologic basis implies that in SPMS patients neurodegeneration occurs from the beginning of disease, even before symptoms of relapses (clinically active inflammation) appear. Likewise, there will be an ongoing chronic "below the radar" subclinical inflammation in PPMS patients, prior to onset of progressive neurodegenerative symptoms. Accordingly, it has been recently proposed that a cascade of immunological and neurodegenerative

Table 1 Key aspects of progressive multiple sclerosis (PMS) distinguishing it from relapsing-remitting multiple sclerosis (RRMS)

\footnotetext{
- Time to disability progression is not driven by relapse rate, frequency or severity

- Fewer active plaques (less inflammation and peripheral immune cell activation and fewer gadolinium enhancing lesions [signifying fewer blood-brain barrier breaches])

- Compartmentalised inflammation within the central nervous system (CNS): meningeal inflammatory aggregates (lymphatic follicles-like)

- More neurodegeneration: more demyelination and axonal loss in gray matter; more cortical pathology (subpial lesions are almost specific of PMS) and CNS atrophy

- More diffuse abnormalities and tissue loss in the normal appearing CNS

- Universal progressive spinal cord disease

- Anti-inflammatory therapies less effective or ineffective
} 
events act in concert to induce MS specific CNS damage [12]. Key elements driving neurodegeneration include microglia activation, chronic oxidative injury, accumulation of mitochondrial damage in axons, and age-related iron accumulation in the human CNS [5, 13, 14, 21, 23, 24]. Altered mitochondrial damage in axons might be of particular importance since it leads to chronic cell stress and imbalance of ionic homeostasis, resulting in axonal and neuronal death.

Loss of the symbiotic relationship between the axon and myelin sheath after persistent demyelination in MS causes progressive axonal damage providing a logical explanation for continuous and irreversible neurological decline in PMS. Chronic deprivation of myelin-derived trophic support of axons, results in a slowly development of axonopathy manifested by axonal swelling, reduction in axonal calibre and ultimately axonal degeneration. Current hypotheses indicate that the compensatory plasticity of the CNS delays the onset and progression of neurological decline in MS patients. Adaptative and neuroprotective mechanisms that may compensate for initial dysfunction include activation of cortical areas, neurogenesis, remyelination, redistribution of sodium channels on demyelinated axons, increases in mitochondrial content and respiratory functions in axons and enhanced production of neurotrophic factors by CNS resident cells [22, 25]. However, once a threshold of axonal loss is reached the exhaustion of the functional reservoir results in a steady progression of MS.

\section{Neuropathological features of progressive multiple sclerosis}

How do chronically demyelinated axons and neurons degenerate? It has been suggested that failure of remyelination is the pathological substrate for disease progression in MS $[1,26]$. In fact, the pattern of tissue injury in PMS is quite homogeneous and it is characterized by oligodendrocyte loss, demyelination and lack of remyelination, as well as preferential destruction of small-calibre axons and astrocytic gliosis. Characteristics of PMS pathology are focal lesions of demyelination, strips or bands of cortical demyelination and diffuse pathology in the normal appearing white and grey matter (Table 1).

- An absence of focal new inflammatory demyelinating lesions as measured by MRI and histopathology is predominant in the course of PMS [2]. Inactive or slowly spanding pre-existing MS plaques are usually seen, latter characterized by a rim of microglia and a low-grade myelin and axonal destruction at the lesion edge. But "inactivity" does not mean stability of disease lesions. Most axons can survive acute demyelination, and some grade of remyelination is possible if active lesions are resolved, but chronically demyelinated axons degenerate. Dynamics of axonal loss are not well documented but this is a prominent feature of PMS that can explain partly the concomitant CNS atrophy observed and is the major cause of irreversible neurological disability.

- Cortical and deep grey matter demyelination is very common in MS and exceeds white matter demyelination. However, little is known about its dynamics because lesion load measures have been difficult both by MRI and by histopathology [26-28]. In PMS strips or bands of subpial cortical demyelination, which differ in appearance from intracortical and leukocortical focal lesions ones, are by far the biggest contributors to total cortical lesion load, and do not occur in other inflammatory or non-inflammatory human CNS diseases. In addition to demyelination and oligodendrocyte loss, cortical lesions show neuritic transections, neuronal death and synaptic loss and when active are linked to local inflammation in the meninges and activation of microglia. Similar alterations also occur in spinal cord and other CNS locations of grey matter.

- On the other hand, macroscopically normal appearing CNS of patients with MS also show diffuse and global changes that are already present from the beginning of disease $[19,21]$. They include microglial activation, astrocytic gliosis and mild inflammation, demyelination, and axonal injury. With time there is an increasing and progressive loss of axons in the normal-appearing white matter and of neurons in gray matter which correlates with the CNS atrophy that appear in advanced PMS. This atrophy does not correlate with focal lesions load suggesting that diffuse neurodegeneration is at least partly, an independent process from anterograde Wallerian degeneration and/or retrograde neuronal dying back started in white and gray plaques.

\section{Molecular mechanisms underlying progression in multiple sclerosis}

They are summarized in Table 2 and detailed in the following subsections.

\section{Inflammation and microglial activation}

In all forms of MS, inflammation is always present when active demyelination and neurodegeneration occur [3, 12, 20]. Inflammatory released products as reactive oxygen or nitric oxide species (ROS and RNS), excitotoxins as glutamate, and cytotoxic cytokines alter myelin sheaths and cellular metabolism in neurons and their axons. However inflammation declines with disease duration while neurodegeneration proceeds and activated microglia persists in all lesions in PMS [29]. 
Table 2 Mechanisms and amplification factors for neurodegeneration in progressive multiple sclerosis (PMS)

- Chronic microglial activation and low-grade inflammation: excitotoxicity, and chronic oxidative stress which leads to mitochondrial injury

- Mitochondrial dysfunction (energy failure; ionic imbalance) that might underlie features of Multiple Sclerosis lesions: demyelination, oligodendrocyte apoptosis and lack of remyelination; and axonal injury

- Accumulation of lesion burden: retrograde and anterograde degeneration of demyelinated axons due to lack of trophic support and abnormal axonal excitability (altered expression of ion channels) and amplification of microglia activation

- Mitochondrial DNA deletion over time and with age as in other classical neurodegenerative diseases: increased energy deficiency and amplification of oxidative injury

- Iron accumulation with ageing in the brain cells and release of iron in demyelinating lesions (more oxidative injury)

- Progression of age-related neurodegeneration and exhaustion of central nervous system functional reserve capacity

Whether inflammation in PMS, as in other stages of disease, is a cause (the outside-in theory: immune dysregulation) or result of neurodegeneration (inside-out model: governed by released immunogenic material after cytodegeneration) remains to be determined [15]. Also, a key unresolved question is if the inflammatory response is qualitatively the same in PMS as in other stages of disease where different patterns of inflammatory processes occur [30, 31]. Quantitatively, PMS inflammation becomes, at least in part, trapped within the CNS behind an unaffected $\mathrm{BBB}$ forming lymph follicle-like aggregates in the meninges. Demyelination and neurodegeneration in the cortex of PMS patients are more pronounced when these structures are present [32].

Microglial activation, probably due to a chronically inflammatory milieu, is invariably seen in the CNS of patients with PMS [33]. However this is common in many other CNS diseases in absence of selective primary demyelination. Perhaps the "specific" contribution of chronically activated microglia to the induction of demyelination and progressive axonal injury in MS is the generation of chronic oxidative stress, by xanthine and NADPH (nicotinamide-adenine dinucleotide phosphate) oxidases as well as myeloperoxidase, and subsequent mitochondrial injury in axons and oligodendrocytes. In addition, activated microglia impairs glutamate transport in astrocytes, and thus, promote neuronal and oligodendrocyte excitotoxicity $[5,12,33]$.

\section{Mitochondrial injury and axonal energy failure}

Several lines of evidence have led to the hypothesis that mitochondrial injury is a primary phenomenon in MS $[24,34]$. Remarkably, mitochondria and mitochondrial DNA, essential for oxidative phosphorylation, are highly susceptible to oxidative injury. In fact, chronic increased local levels of ROS and RNS by activated microglia are pronounced in PMS despite low levels of inflammation and can promote mitochondrial dysfunction. Moreover, the mechanisms of axonal degeneration in MS are similar to those occurring in ischemic/hypoxic insults. Thus, it has been hypothesized that energy deficiency or "virtual hypoxia" might have also a pathogenic role in MS. Demyelination has a substantial effect on axonal mitochondria and renders the demyelinated axon susceptible to chronic environmental conditions that eventually result in axon transection and degeneration. Key features related to mitochondrial dysfunction $[2,5,12,13,19,22,34]$ are the following:

- After demyelination, aberrant expression of $\mathrm{Na}^{+}$ channels along the entire length of the demyelinated axon increases energy demands of nerve conduction, and ATP production by mitochondria might become compromised causing axoplasmic ionic imbalance and intracellular $\mathrm{Ca}^{2+}$ accumulation. In conditions of energy deficiency and mitochondrial "functional overload", the ATP-dependent $\mathrm{Na}^{+}$pump does not remove $\mathrm{Na}^{+}$, which accumulates in the axon an is replaced by $\mathrm{Ca}^{2+}$ through reverse operation of the $\mathrm{Na}^{+} / \mathrm{Ca}^{2+}$ exchanger. Additional $\mathrm{Ca}^{2+}$ enters the axon via glutamate receptors and voltage-gated $\mathrm{Ca}^{2+}$ channels present in the axolemma. If mitochondrial damage passes a certain threshold, reduced local energy production might precipitate axonal demise. In that way, thin-calibre axons with proportionally less mitochondria in relation to the surface area of axolemma than thick ones, are more severely affected.

- When axoplasmic $\mathrm{Ca}^{2+}$ accumulation exceeds the buffering capacity by mitochondria, initiates a vicious cycle of deleterious and finally fatal effects on the axon characterized by activation of degradative enzymes that compromise axonal transport, impaired mitochondrial operation, reduced energy production, and more axoplasmic $\mathrm{Ca}^{2+}$. In fact, cytoskeletal disturbances as fragmentation of neurofilaments by $\mathrm{Ca}^{2+}$ activated degradative enzymes as calpain, appear in chronic MS lesions, altering the turnover and redistribution of mitochondria and fast axonal transport. Also, in patients with PMS, a lost or impaired functional activity of mitochondrial respiratory chain complexes has been described in neurons. Therefore, chronic mitochondrial injury amplifies chronic oxidative stress present in MS and viceversa. Additionally, oxidative injury causes mitochondrial DNA deletions in both the white matter and grey matter. Accumulation of mitochondrial dysfunctions in patients with PMS as a final result of the 
demyelination, could partly explain their increased susceptibility of brain tissue to neurodegeneration through enhancement of oxidative injury, energy failure and altered calcium homeostasis.

- In oligodendrocytes, mitochondrial injury results in the release of apoptosis-inducing factor, its translocation into the nucleus and induction of DNA damage which ultimately leads to cell death.

\section{Age-dependent iron accumulation amplifies injury in PMS}

Iron accumulation in patients with MS can further amplify ROS and RNS mediated injury, by generating toxic reactants $[2,5,12]$. Iron loading in the brain increases with age and is predominantly stored in oligodendrocytes in the non-toxic ferric $\left(\mathrm{Fe}^{3+}\right)$ form bound to ferritin. Importantly, injury of these cells during MS releases iron into the extracellular space where it is converted into $\mathrm{Fe}^{2+}$, which might further amplify oxidative damage in axons and other cells. Released iron is then taken up by activated microglia, which becomes dystrophic and degenerate, releasing a second way of $\mathrm{Fe}^{2+}$ and increasing the susceptibility of the surrounding tissue to free-radical-driven demyelination and neurodegeneration. It is worth noting that although iron accumulation is an age-dependent process, and for that reason likely more pronounced in PMS than previously in the disease, the pathogenic role of iron in MS is on discussion. In fact, in chronic MS, a significant decrease in iron levels is observed in normal appearing CNS [35]. Also it must be taken into account that previous disease history also influences the development of neurodegenerative amplification damage. In fact, the earlier and/or higher relapse rates, the shorter time to onset of PMS [9].

\section{Advances in imaging in progressive multiple sclerosis}

Pathological assessment is the gold standard to identify MS lesions, but there are intrinsic limitations due to the very limited availability of biopsy tissue and additionally, tissue evaluation only provides one snapshot in time, not allowing observation of the evolution of pathological changes over time. Because of that, MRI and related techniques with higher specificity are promising tools for a better understanding of the pathophysiology of the PMS “in vivo" [8]. Although conventional T1-weighted images show less sensitivity than T2-weighted MRI images, they are more specific to MS pathology and probably $\mathrm{T} 1$ hypointense signals are reflecting tissue lesions with oedema, demyelination, neuroaxonal loss and gliosis [36]. In clinical practice, it is difficult to differentiate a case of PMS from RRMS using MRI [8, 37-39], but studies have found some population-based differences between these two groups. In PMS, there is somehow a preponderance of spinal cord/brainstem lesions and relative paucity of new gadolinium-enhancing brain lesions over time. A decrease in their occurrence is related to the clinical transition from RRMS to SPMS. Also there are differences in brain atrophy localization, mainly due to loss of white matter in RRMS as seen by ventricular enlargement, while in PMS atrophy tends to be more a cortical phenomenon, particularly common in PPMS. However conventional MRI approaches are basically unable to detect cortical lesions and diffuse tissue damage in the normal appearing CNS. As a consequence, they correlate only weakly with clinical manifestations and evolution of PMS.

On the contrary, advanced MRI techniques that are far away of being integrated into routine clinical practice at present, have shown greater sensitivity and specificity for assessing the pathological substrates of MS, in particular demyelination and neuroaxonal loss, providing better prognostic information [13, 40-42]. These include magnetization transfer (MT) imaging and its quantitative index MT ratio which has been proposed as a marker of brain myelin content, also in the cerebral cortex; diffusion tensor MRI tractography for axonal loss; and proton MR spectroscopy that records signals from metabolites as $\mathrm{N}$-acetyl aspartate, a marker of neuroaxonal integrity, or choline and lactate, biochemical correlates of inflammation and demyelination. Additional insights into the characteristics of MS lesions have been obtained from iron-sensitive MRI sequences, especially at very high field strengths (7.0 Tesla) where its accumulation shows a close match to neurodegeneration. These quantitative MR techniques by measuring the disease burden within focal lesions and in the normal-appearing CNS instead volume changes are contributing significantly to the understanding of the pathological mechanisms underlying the irreversible accumulation of disability in PMS. However, and despite their potential, most of the new MR techniques still require future research and validation.

\section{Potential therapies}

Oligodendrocytes and axons express neurotransmitter receptors to glutamate so it may act as a potential excitotoxin under acute and chronic insults, and ultimately contribute to neurodegeneration in PMS. Thus, numerous studies conducted in cellular and animal models of MS, as well as in post-mortem brain and in patients, indicate that excitotoxicity mediated by $\mathrm{Ca}^{2+}$-permeable glutamate receptors contributes to oligodendrocyte death, demyelination, and tissue damage in MS [43]. In particular, experimental MS is alleviated by $\alpha$-amino-3-hydroxy-5methyl-4-isoxazolepropionate (AMPA) and kainate receptor antagonists, but not N-methyl-d-aspartate receptor blockade, and combination with anti-inflammatory agents expands neuroprotection even at advanced disease stages [44]. Moreover, genome-wide association screening 
associated alleles in AMPA receptor genes in MS patients to brain volume loss in patients with high glutamate levels [45]. In turn, glutamate levels are increased in the brain in MS as a consequence of reduced expression of the glutamate transporters (excitatory amino acid transporters 1 and 2) and upregulation of the cystine/glutamate antiporter in the monocyte-macrophage-microglia lineage is associated with immune activation in both MS and experimental autoimmune encephalomyelitis (EAE) [43, 46]. Like glutamate, ATP, when in excess, is a potent endogenous toxin that can directly kill oligodendrocytes via activation of purinergic P2X7 receptors whose blockade during the chronic phase of EAE attenuates the symptoms and tissue damage [47]. Interestingly, P2X7 variants are associated with a reduced or increased risk to suffer from MS $[48,49]$. All in all, these studies in MS animal models and in the disease proper offer new ideas to develop novel therapies to treat PMS.

Recently, results of clinical trials have challenged the long-held belief that pathogenesis of PMS is more neurodegenerative than inflammatory, and distinct from these aspects in RRMS. Indeed, ocrelizumab, a humanized monoclonal antibody targeting CD20 B cells, has significant efficacy in both forms of MS reducing the relapse rate in RRMS and delaying the risk of clinical disability progression in RRMS and, although modestly, also for the first time in primary PMS [50]. Pivotal ocrelizumab trials $[51,52]$ had showed that mature B cells, which express the antigen CD20 on their surface, played a central role in the pathophysiology of RRMS traditionally considered to be driven by $\mathrm{T}$ cells due to the observations of activated $\mathrm{T}$ lymphocytes in MS plaques $[2,3,6,19]$. This idea is now extended to PMS where lymphoid follicles containing $B$ cells are present in the meninges [32]. Although the mechanisms whereby anti-CD20 exerts its beneficial effects are not well understood, there is evidence that CD20 B cells, which represent a few percent of the total B-cell pool, are involved in progressive aspects of MS [53]. Moreover, clinical trials have shown that CD20 B-cell depletion is effective without significant compromise of the normal immune reactivity. Further stratification of disease subtypes will be needed for a better understanding of the complex roles of B cells in MS [54-56]. Also, if the presence of disease activity measured as $\mathrm{T} 1$ gadoliniumenhancing lesions favours the response to ocrelizumab as suggested by recent data [57]. However, the lack of biomarkers predicting the level of activity and/or progression in MS, namely active demyelination and neurodegeneration, hinders the stratification of disease types $[11,18]$. In this regard, further promising developments in PMS therapies with targeted disease-modifying drugs may contribute to better understanding the underlying molecular and cellular pathology of MS. Lastly, but not least, global research collaboration will be needed to overcome the challenges of understanding and treating efficiently PMS $[23,58]$.

\section{Conclusions}

In MS, the progression of disease is the consequence of a pathogenic cascade of events caused and/or related to long-lasting accumulation of CNS damage which initiated earlier, even when clinical symptoms were not present. Chronic inflammation and neurodegeneration are interlinked in MS from early stages of disease course and there are multiple potential mechanisms that trigger and sustain damage in PMS $[59,60]$. Oxidative damage, severe ion channel dysfunction, mitochondrial injury, microglial activation without obvious disruption of the $\mathrm{BBB}$, and age related changes in CNS, are mechanisms that also contribute to other neurodegenerative diseases. Unlike in other neurodegenerative conditions, there is no evidence of a primary trigger in MS for myelin and oligodendrocytic injury, and it is unknown why usually it has not a monophasic course from its beginning. Perhaps the absence of remission in PMS is a sign of exhaustion of CNS compensatory mechanisms rather than a change in the disease process. Moreover, there is no imaging, pathological, or biomarker characteristics that reliably distinguish between first episodes of MS and progressive MS. Although the molecular and cellular mechanisms driving the cyclic course and/or progression of disease are incompletely understood, widespread and diffuse loss of neurons and axons in the white and grey matter with resultant atrophy has a key role in disability progression in MS. In fact, subpial cortical demyelination appears to be exquisitely specific for MS, since it is not present in any other inflammatory, neurodegenerative or metabolic CNS disease. Despite the unknowns, from a therapeutic point of view, a combination of immunomodulatory and neuroprotective strategies introduced earlier in the development of disease appear to be the most promising strategy to limit the progression of MS. Also appropriate animal models, good biomarkers and imaging measures remain indispensable to understand the disease and test novel potential treatments.

\section{Abbreviations \\ AMPA, a-amino-3-hydroxy-5-methyl-4-isoxazolepropionate; ATP, Adenosine triphosphate; BBB, Blood brain barrier; $\mathrm{Ca}^{2+}$, Calcium ion; CNS, Central nervous system; DNA, Deoxyribonucleic acid; EAE, Experimental autoimmune encephalomyelitis; $\mathrm{Fe}^{2+} ; \mathrm{Fe}^{3+}$, Ferrous ion; Ferric ion; MRI, Magnetic resonance imaging; MS, Multiple sclerosis; $\mathrm{Na}^{+}$, Sodium ion; $\mathrm{NADPH}$, Nicotinamide-adenine dinucleotide phosphate (reduced form); PMS, Progressive multiple sclerosis; PPMS, Primary progressive multiple sclerosis; RNS, Reactive nitric oxide species; ROS, Reactive oxygen species; RRMS, Relapsing-remitting multiple sclerosis; SPMS, Secondary progressive multiple sclerosis}

\section{Acknowledgements}

Work in our laboratory is funded by CIBERNED, Gobierno Vasco (EJ/GV) and MINECO (SAF2013-45084-R). 


\section{Authors' contributions}

FP-C designed and coordinated the review and drafted the manuscript. MVS-G revised it critically for important intellectual content. CM conceived the review, participated in its design and coordination and helped to draft the manuscript. All authors read and approved the final manuscript.

\section{Competing interests}

The authors declare that they have no competing interests.

\section{Search strategy and selection criteria}

We searched PubMed with the terms "progressive multiple sclerosis", "magnetic resonance imaging" and "clinical trials" in papers published in English. References were also identified through searches of unloaded articles and of our own files. We focused on the previous decade of research and on reviews with a great influence in the understanding of PMS. Final list of publications was selected on the basis of relevance to the aims of this review.

Received: 23 December 2015 Accepted: 24 May 2016

Published online: 07 July 2016

\section{References}

1. Trapp BD, Nave KA. Multiple sclerosis: an immune or neurodegenerative disorder? Annu Rev Neurosci. 2008:31:247-69.

2. Lassmann H, van Horssen J, Mahad D. Progressive multiple sclerosis: pathology and pathogenesis. Nat Rev Neurol. 2012:8:647-56.

3. Stadelmann C, Wegner C, Brück W. Inflammation, demyelination, and degeneration - recent insights from MS pathology. Biochim Biophys Acta. 1812:2011:275-82

4. Ellwardt E, Zipp F. Molecular mechanisms linking neuroinflammation and neurodegeneration in MS. Exp Neurol. 2014;262:8-17.

5. Friese MA, Schattling B, Fugger L. Mechanisms of neurodegeneration and axonal dysfunction in multiple sclerosis. Nat Rev Neurol. 2014;10:225-38.

6. Kutzelnigg A, Lassmann H. Pathology of multiple sclerosis and related inflammatory demyelinating diseases. In: Goodin DS, editor. Handbook of clinical neurology (vol 122): Multiple sclerosis and related disorders. Amsterdam: Elsevier; 2014. p. 15-58.

7. Schaeffer J, Cossetti C, Mallucci G, Pluchino S. Multiple sclerosis. In: Zigmond MJ, Rowland LP, Coyle JT, editors. Neurobiology of brain disorders: biological basis of neurological and psychiatric disorders. London: Academic; 2015. p. 497-520.

8. Polman CH, Reingold SC, Banwell B, Clanet M, Cohen JA, Filippi M, et al. Diagnostic criteria for multiple sclerosis: 2010 revisions to the McDonald criteria. Ann Neurol. 2011:69:292-302.

9. Lublin FD, Reingold SC, Cohen JA, Cutter GR, Sørensen PS, Thompson AJ, et al. Defining the clinical course of multiple sclerosis: the 2013 revisions. Neurology. 2014;83:278-86.

10. Ontaneda D, Fox RJ. Progressive multiple sclerosis. Curr Opin Neurol. 2015;28:237-43

11. Ontaneda D, Fox RJ, Chataway J. Clinical trials in progressive multiple sclerosis: lessons learned and future perspectives. Lancet Neurol. 2015;14:208-23.

12. Mahad DH, Trapp BD, Lassmann H. Pathological mechanisms in progressive multiple sclerosis. Lancet Neurol. 2015;14:183-93.

13. Ciccarelli O, Barkhof F, Bodini B, De Stefano N, Golay X, Nicolay K, et al. Pathogenesis of multiple sclerosis: insights from molecular and metabolic imaging. Lancet Neurol. 2014;13:807-22.

14. Haider L, Simeonidou C, Steinberger G, Hametner S, Grigoriadis N, Deretzi G, et al. Multiple sclerosis deep grey matter: the relation between demyelination, neurodegeneration, inflammation and iron. J Neurol Neurosurg Psychiatry. 2014;85:1386-95.

15. Stys PK, Zamponi GW, van Minnen J, Geurts JJ. Will the real multiple sclerosis please stand up? Nat Rev Neurosci. 2012;13:507-14.

16. Kamm CP, Uitdehaag BM, Polman CH. Multiple sclerosis: current knowledge and future outlook. Eur Neurol. 2014;72:132-41.

17. Feinstein A, Freeman J, Lo AC. Treatment of progressive multiple sclerosis: what works, what does not, and what is needed. Lancet Neurol. 2015;14:194-207.

18. Koch MW, Cutter G, Stys PK, Yong WW, Metz LM. Treatment trials in progressive MS -current challenges and future directions. Nat Rev Neurol. 2013;9:496-503.
19. Dutta R, Trapp BD. Relapsing and progressive forms of multiple sclerosis: insights from pathology. Curr Opin Neurol. 2014;27:271-8.

20. Calabrese M, Magliozzi R, Ciccarelli O, Geurts JJ, Reynolds R, Martin R. Exploring the origins of grey matter damage in multiple sclerosis. Nat Rev Neurosci. 2015;16:147-58.

21. Lassmann H. Multiple sclerosis: Lessons from molecular neuropathology. Exp Neurol. 2014;262:2-7.

22. Dutta R, Trapp BD. Mechanisms of neuronal dysfunction and degeneration in multiple sclerosis. Prog Neurobiol. 2011;93:1-12.

23. Salvetti M, Landsman D, Schwarz-Lam P, Comi G, Thompson AJ, Fox RJ. Progressive MS: from pathophysiology to drug discovery. Mult Scler 2015;21:1376-84.

24. Criste G, Trapp B, Dutta R. Axonal loss in multiple sclerosis: causes and mechanisms. In: Goodin DS, editor. Handbook of clinical neurology (vol 122): Multiple sclerosis and related disorders. Amsterdam: Elsevier; 2014. p. 101-13.

25. Bramow S, Frischer JM, Lassmann H, Koch-Henriksen N, Lucchinetti CF, Sørensen PS, et al. Demyelination versus remyelination in progressive multiple sclerosis. Brain. 2010;133:2983-98.

26. Lubetzki C, Stankoff B. Demyelination in multiple sclerosis. In: Goodin DS, editor. Handbook of clinical neurology (vol 122): Multiple sclerosis and related disorders. Amsterdam: Elsevier; 2014. p. 89-99.

27. Prins M, Schul E, Geurts J, van der Valk P, Drukarch B, van Dam AM. Pathological differences between white and grey matter multiple sclerosis lesions. Ann N Y Acad Sci. 2015;1351:99-113.

28. Fischer MT, Wimmer I, Höftberger R, Gerlach S, Haider L, Zrzavy T, et al. Disease-specific molecular events in cortical multiple sclerosis lesions. Brain. 2013:136:1799-815.

29. Frischer JM, Weigand SD, Guo Y, Kale N, Parisi JE, Pirko I, et al. Clinical and pathological insights into the dynamic nature of the white matter multiple sclerosis plaque. Ann Neurol. 2015;78:710-21.

30. Lassmann H. Pathology and disease mechanisms in different stages of multiple sclerosis. J Neurol Sci. 2013;333:1-4

31. Metz I, Weigand SD, Popescu BF, Frischer JM, Parisi JE, Guo Y, et al. Pathologic heterogeneity persists in early active multiple sclerosis lesions. Ann Neurol. 2014:75:728-38.

32. Absinta M, Vuolo L, Rao A, Nair G, Sati P, Cortese IC, et al. Gadolinium-based MRI characterization of leptomeningeal inflammation in multiple sclerosis. Neurology. 2015;85:18-28.

33. Correale J. The role of microglial activation in disease progression. Mult Scler. 2014;20:1288-95.

34. Campbell GR, Worrall JT, Mahad DJ. The central role of mitochondria in axonal degeneration in multiple sclerosis. Mult Scler. 2014;20:1806-13.

35. Hametner S, Wimmer I, Haider L, Pfeifenbring S, Brück W, Lassmann H. Iron and neurodegenration in the multiple sclerosis brain. Ann Neurol. 2013;74:848-61.

36. Filippi M, Rocca MA, Barkhof F, Brück W, Chen JT, Comi G, et al. Association between pathological and MRI findings in multiple sclerosis. Lancet Neurol. 2012;11:349-60.

37. Rovira A, Wattjes MP, Tintoré M, Tur C, Yousry TA, Sormani MP, et al. Evidence-based guidelines: MAGNIMS consensus guidelines on the use of $\mathrm{MRI}$ in multiple sclerosis-clinical implementation in the diagnostic process. Nat Rev Neurol. 2015:11:471-82.

38. Wattjes MP, Rovira A, Miller D, Yousry TA, Sormani MP, de Stefano MP, et al. Evidence-based guidelines: MAGNIMS consensus guidelines on the use of MRI in multiple sclerosis-establishing disease prognosis and monitoring patients. Nat Rev Neurol. 2015;11:597-606.

39. Filippi M, Rocca MA, Ciccarelli O, De Stefano N, Evangelou N, Kappos L, et al. MRI criteria for the diagnosis of multiple sclerosis: MAGNIMS consensus guidelines. Lancet Neurol. 2016;15:292-303.

40. Filippi M, Rocca MA, De Stefano N, Enzinger C, Fisher E, Horsfield MA, et al. Magnetic resonance techniques in multiple sclerosis: the present and the future. Arch Neurol. 2011;68:1514-20.

41. Rocca MA, Absinta M, Filippi M. The role of advanced magnetic resonance imaging techniques in primary progressive MS. J Neurol. 2012:259:611-21.

42. Enzinger C, Barkhof F, Ciccarelli O, Filippi M, Kappos L, Rocca MA, et al. Nonconventional MRI and microstructural cerebral changes in multiple sclerosis. Nat Rev Neurol. 2015;11:676-86.

43. Butt AM, Fern RF, Matute C. Neurotransmitter signaling in white matter. Glia. 2014;62:1762-79. 
44. Kanwar JR, Kanwar RK, Krissansen GW. Simultaneous neuroprotection and blockade of inflammation reverses autoimmune encephalomyelitis. Brain. 2004;127:1313-31.

45. Baranzini SE, Srinivasan R, Khankhanian P, Okuda DT, Nelson SJ, Matthews PM, et al. Genetic variation influences glutamate concentrations in brains of patients with multiple sclerosis. Brain. 2010;133:2603-11.

46. Pampliega O, Domercq M, Soria FN, Villoslada P, Rodríguez-Antigüedad A, Matute C. Increased expression of cystine/glutamate antiporter in multiple sclerosis. J Neuroinflammation. 2011;8:63.

47. Matute C, Torre I, Pérez-Cerdá F, Pérez-Samartín A, Alberdi E, Etxebarria E, et al. P2X(7) receptor blockade prevents ATP excitotoxicity in oligodendrocytes and ameliorates experimental autoimmune encephalomyelitis. J Neurosci. 2007;27:9525-33.

48. Oyanguren-Desez O, Rodríguez-Antigüedad A, Villoslada P, Domercq M, Alberdi $\mathrm{E}$, Matute C. Gain-of-function of P2X7 receptor gene variants in multiple sclerosis. Cell Calcium. 2011;50:468-72.

49. Gu BJ, Field J, Dutertre S, Ou A, Kilpatrick TJ, Lechner-Scott J, et al. A rare P2X7 variant Arg307GIn with absent pore formation function protects against neuroinflammation in multiple sclerosis. Hum Mol Genet. 2015;24:5644-54.

50. Montalban X, Hemmer B, Rammohan K, Giovannoni G, de Seze J, Bar-Or A, et al. Efficacy and safety of ocrelizumab in primary progressive multiple sclerosis-results of the placebo-controlled, double-blind, Phase III ORATORIO study. Mult Scler. 2016;22(S1):17.

51. Kappos L, Li D, Calabresi PA, O'Connor P, Bar-Or A, Barkhof F, et al. Ocrelizumab in relapsing-remitting multiple sclerosis: a phase 2, randomised, placebo-controlled, multicentre trial. Lancet. 2011;378:1779-87.

52. Chataway J, Miller DH. Multiple sclerosis-quenching the flames of inflammation. Lancet. 2011;378:1759-60.

53. Sorensen PS, Blinkenberg M. The potential role for ocrelizumab in the treatment of multiple sclerosis: current evidence and future prospects. Ther Adv Neurol Disord. 2016;9:44-52.

54. Steinman L, Zamvil SS. Beginning of the end of two-stage theory purporting that inflammation then degeneration explains pathogenesis of progressive multiple sclerosis. Curr Opin Neurol. 2016;29: Epub ahead of print. doi:10.1097/WCO.0000000000000317

55. Sheridan C. Anti-CD20 antibody wows in multiple sclerosis. Nat Biotechnol. 2015:33:1215-6.

56. Milo R. Therapeutic strategies targeting B-cells in multiple sclerosis. Autoimmun Rev. 2016; doi:10.1016/j.autrev.2016.03.006

57. Wolinsky JS, Arnold D, Bar-Or A, de Seze J, Giovannoni G, Hemmer B, et al. Efficacy of ocrelizumab in patients with PPMS with and without T1 gadolinium-enhancing lesions at baseline in a Phase III, placebo-controlled trial. Mult Scler. 2016;22(S1):67-8.

58. Shirani A, Okuda DT, Stüve O. Therapeutic advances and future prospects in progressive forms of multiple sclerosis. Neurotherapeutics. 2016;13:58-69.

59. Hutchinson M. Neurodegeneration in multiple sclerosis is a process separate from inflammation: No. Mult Scler. 2015;21:1628-31.

60. Louapre C, Lubetzki C. Neurodegeneration in multiple sclerosis is a process separate from inflammation: Yes. Mult Scler. 2015;21:1626-8.

\section{Submit your next manuscript to BioMed Central and we will help you at every step:}

- We accept pre-submission inquiries

- Our selector tool helps you to find the most relevant journal

- We provide round the clock customer support

- Convenient online submission

- Thorough peer review

- Inclusion in PubMed and all major indexing services

- Maximum visibility for your research

Submit your manuscript at www.biomedcentral.com/submit 